\title{
Making Sense of Emotion-Sensing: Workshop on Quantifying Human Emotions
}

\author{
Benjamin Tag \\ benjamin.tag@unimelb.edu.au \\ The University of Melbourne \\ Australia \\ Vanessa Bartlett \\ vanessa.bartlett@unimelb.edu.au \\ The University of Melbourne \\ Australia \\ Petr Slovak \\ petr.slovak@kcl.ac.uk \\ King's College London \\ United Kingdom
}

\author{
Sarah Webber \\ s.webber@unimelb.edu.au \\ The University of Melbourne \\ Australia \\ Jorge Goncalves \\ jorge.goncalves@unimelb.edu.au \\ The University of Melbourne \\ Australia \\ Wally Smith \\ wsmith@unimelb.edu.au \\ The University of Melbourne \\ Australia
}

\author{
Greg Wadley \\ greg.wadley@unimelb.edu.au \\ The University of Melbourne \\ Australia \\ Peter Koval \\ p.koval@unimelb.edu.au \\ The University of Melbourne \\ Australia \\ Tom Hollenstein \\ tom.hollenstein@queensu.ca \\ Queen's University \\ Canada
}

\author{
Anna Cox \\ anna.cox@ucl.ac.uk \\ University College London \\ United Kingdom
}

\author{
Vassilis Kostakos \\ vassilis.kostakos@unimelb.edu.au \\ The University of Melbourne \\ Australia
}

\begin{abstract}
The global pandemic and the uncertainty if and when life will return to normality have motivated a series of studies on human mental health. This research has elicited evidence for increasing numbers of anxiety, depression, and overall impaired mental well-being. But, the global COVID-19 pandemic has also created new opportunities for research into quantifying human emotions: remotely, contactless, in everyday life. The ubiquitous computing community has long been at the forefront of developing, testing, and building userfacing systems that aim at quantifying human emotion. However, rather than aiming at more accurate sensing algorithms, it is time to critically evaluate whether it is actually possible and in what ways it could be beneficial for technologies to be able to detect user emotions. In this workshop, we bring together experts from the fields of Ubiquitous Computing, Human-Computer Interaction, and Psychology to - long-overdue - merge their expertise and ask the fundamental questions: how do we make sense of emotion-sensing, can and should we quantify human emotions?
\end{abstract}

\section{CCS CONCEPTS}

- Human-centered computing $\rightarrow$ Human computer interaction (HCI); Ubiquitous and mobile computing; • Applied computing $\rightarrow$ Psychology.

Permission to make digital or hard copies of all or part of this work for personal or classroom use is granted without fee provided that copies are not made or distributed for profit or commercial advantage and that copies bear this notice and the full citation on the first page. Copyrights for components of this work owned by others than the author(s) must be honored. Abstracting with credit is permitted. To copy otherwise, or republish, to post on servers or to redistribute to lists, requires prior specific permission and/or a fee. Request permissions from permissions@acm.org.

UbiComp-ISWC '21 Adjunct, September 21-26, 2021, Virtual, USA

(c) 2021 Copyright held by the owner/author(s). Publication rights licensed to ACM. ACM ISBN 978-1-4503-8461-2/21/09...\$15.00

https://doi.org/10.1145/3460418.3479272

\section{KEYWORDS}

Emotions; Emotion Detection; Sensing; Ubiquitous Computing

ACM Reference Format:

Benjamin Tag, Sarah Webber, Greg Wadley, Vanessa Bartlett, Jorge Goncalves, Peter Koval, Petr Slovak, Wally Smith, Tom Hollenstein, Anna Cox, and Vassilis Kostakos. 2021. Making Sense of Emotion-Sensing: Workshop on Quantifying Human Emotions. In Adjunct Proceedings of the 2021 ACM International foint Conference on Pervasive and Ubiquitous Computing and Proceedings of the 2021 ACM International Symposium on Wearable Computers (UbiComp-ISWC '21 Adjunct), September 21-26, 2021, Virtual, USA. ACM, New York, NY, USA, 4 pages. https://doi.org/10.1145/3460418.3479272

\section{INTRODUCTION}

The uncertainty during the COVID-19 pandemic and the consequently extensive restrictions to our regular lifestyles have caused a global mental health crisis $[6,26]$. Despite the obvious causes, such as the fear of the disease, social distancing measures, potential fatal consequences, financial hardship, and limitations to free movement, interruptions to existing mental health services have been reported in $93 \%$ of countries [25]. The resulting higher demand in mental health support is projected to double the global emotion detection and recognition market in size by $2026^{1}$.

Recent developments in Machine Learning, Artificial Intelligence, and smart technologies have enabled new approaches to quantifying, assessing, and regulating human emotions. However, while some researchers call for making better use of available (ubiquitous) technologies to research human emotions [7, 11], others have raised concerns regarding the potential of emotion detection technologies $[3,8,15]$.

\footnotetext{
${ }^{1}$ https://www.marketsandmarkets.com/Market-Reports/emotion-detectionrecognition-market-23376176.html, last accessed March 28, 2021.
} 
This workshop aims at bringing together experts from the fields of ubiquitous computing (ubicomp), who focus on novel sensing methods and technologies, and experts in psychological research on emotion, emotion detection, and emotion regulation. We will provide a forum to explore new avenues for utilizing existing technologies in emotion research as well as to discuss issues around the implications and intent of quantifying human emotion.

\section{BACKGROUND}

A substantial body of ubicomp research explores the detection of emotion and mood using pervasive, mobile, and wearable sensing solutions. Despite some promising results, many studies highlight the challenges of reliably inferring emotion from autonomous physiological and neural measures such as Electrocardiography (ECG) [9], Electrodermal activity (EDA) (or Galvanic Skin Response (GSR) $[1,10]$, Heart-rate variability (HRV) [16], and Electroencephalography (EEG) $[12,17]$. Similarly, automated detection of facial expressions - though of increasing commercial interest as an approach to infer emotion - is found to be problematic when using naturalistic images [27].

Many emotion detection techniques rely on capturing sensor data relating to four of the five components of emotion commonly identified by psychologists: physiology, expression, behaviour or neural activity. Emotional experience, often referred to as the fifth component $[8,15]$, may be captured through self-report techniques such as Ecological Momentary Assessment [5]. Delplanque and Sander [8] argue that while an expressive signal, such as a smile, or an autonomous physiological measure, such as heart-rate variation, may correlate with specific emotions, it is risky to attempt to infer emotions from measurements of single components. For example, smiling may be correlated with happiness and heart-rate change may be correlated with fear, but these associations between emotions and such measures are "many-to-one" relationships [8]. That is to say, such signals should not be taken as "markers" of specific emotions as there are numerous emotions and non-emotional psychological phenomena that impact any such measures. Furthermore, a facial expression such as a smile or scowl may communicate something other than emotions [3]. Taking measures of multiple components of emotion may provide a more reliable foundation for inferring emotion [8], as is attempted by several recent ubicomp solutions $[4,13,14]$. In such work it is important to keep sight of the subjective, experiential component of emotion. Indeed, Lieberman argues that the felt emotional experience is emotion, rather than being a component of emotion. For, while physiological, behavioural or expressive signals may be detected in the absence of emotion, it is the subjective experience that allows us to definitively say whether an emotion is present or not [15].

The way that human emotion is conceptualised must be examined rigorously as part of efforts to sense and quantify emotion [21]. The theory of constructed emotion [2] proposes that emotional categories are continuously generated by the human brain, rather than existing as universal categories. This presents a challenge to the notion that emotion is something that can be detected and measured.

\subsection{Objectives and Impact}

Emotion-sensing has long been in the focus of Human-Computer Interaction (HCI) and ubicomp research - even giving rise to a new research field: Affective Computing [18]. Recent changes in consumer behavior and technological progress have created new opportunities for research and for the application of emotion detection technology in everyday life, e.g., emotion detection from smartphone usage behavior [19, 20, 24]. But, critical voices questioning the actual ability to detection emotion have become more prominent.

The workshop panel consists of experts in ubicomp (Vassilis Kostakos, Jorge Goncalves, Benjamin Tag), HCI (Anna Cox, Greg Wadley, Wally Smith, Petr Slovak, Sarah Webber), Psychology (Tom Hollenstein, Peter Koval), and the Arts (Vanessa Bartlett). Together with the participants, we aim at developing a new research agenda that is informed by the long history and deep insights into human emotion provided by Psychology, and that takes advantage of the latest developments and findings in HCI.

\section{ORGANIZERS}

Benjamin Tag is a Postdoctoral Research Fellow at the School of Computing and Information Systems at the University of Melbourne. His research focuses on the conceptualisation of digital emotion regulation, and the investigation of human cognition using biometric sensors and psychological test methods. Benjamin has been a main organizer of the workshop series on Eyewear Computing $[22,23]$.

Sarah Webber is a Postdoctoral Research Fellow at the School of Computing and Information Systems at the University of Melbourne. Her research interests include the design of digital technologies for social connectedness and wellbeing.

Greg Wadley is a Senior Lecturer at the School of Computing and Information Systems at the University of Melbourne. He works in Human-Computer Interaction, specializing in health, wellbeing, and emotion. He has worked with psychologists and health researchers to design and evaluate digital interventions for a range of people and conditions. He co-organised a series of $\mathrm{CHI}$ symposia on computing and mental health.

Vanessa Bartlett is a McKenzie Postdoctoral Research Fellow in the School of Culture and Communication at the University of Melbourne. She is interested in ways that artists and curators reimagine technological and networked lives, particularly in relation to wellbeing, agency, and ethics. Her work explores how arts-led research can support understanding of emotion and affect in a digital age.

Jorge Goncalves is a Senior Lecturer at the School of Computing and Information Systems in the University of Melbourne. His research interests include Ubiquitous Computing, Social Computing, Affective Computing, and mobile sensing. He has co-organised several sensing-related workshops at $\mathrm{CHI}$ and UbiComp, and was workshop co-chair for CHI'20 and CHI'21.

Peter Koval is a Senior Lecturer in the Melbourne School of Psychological Sciences at the University of Melbourne. His research focuses on the dynamics of subjective emotional experience and the deliberate regulation of emotion in daily life. 
Petr Slovak is a UKRI Future Leaders Fellow and a Lecturer at King's College London, based at the Informatics and ChildAdolescent Psychiatry department. His research focuses on envisioning, designing, and evaluating new technology-enabled mental health interventions for children and families, with specific focus on emotion regulation.

Wally Smith is an Associate Professor in the School of Computing and Information Systems at the University of Melbourne. His research focus is Human-Centred Computing, with current projects on emotion regulation, deceptive tendencies of $\mathrm{AI}$, and digital engagement in cultural institutions.

Tom Hollenstein is a Professor of Psychology at Queen's University in Kingston, Ontario in Canada and Chair of the Developmental Program. His research focuses on the development and dynamics of emotion regulation across adolescence and into early adulthood. $\mathrm{He}$ is currently bringing his observational, psychophysiological, and interpersonal expertise to bear on the socioemotional development of youth in digital contexts.

Anna Cox is a Professor in the UCL Interaction Centre at University College London. Her research focuses on the relationships between the design of information and communications technologies (ICTs) and behavioural outcomes, and leverages these relationships in the design of novel interfaces and systems to support people in managing their work and wellbeing.

Vassilis Kostakos is a Professor of Computer Science at the University of Melbourne in Australia and Head of the HumanComputer Interaction Group. His research interests focus on Ubiquitous Computing, Human-Computer Interaction, Social Computing, and Internet of Things.

\section{PRE-WORKSHOP}

This workshop is the first of a planned series aiming at developing an interdisciplinary research community within the ubiquitous computing domain. We specifically invite research contributions from Psychology and Cognitive Science.

In preparation of the workshop, we will distribute a Call for Participation and the submission of position papers in UbiComp, Psychology, and Cognitive Science mailing lists through the organizers' networks. We will create a small program committee consisting of the workshop organizers who will peer-review and curate all submissions. This will ensure a targeted but divers workshop program. We will further create a website for the workshop, on which we will announce the workshop program and Call for Participation, introduce the committee members, keynote speakers, and will collate all accepted workshop submissions.

\section{THEMES}

We are proposing the following themes to authors and contributors:

- Quantifying emotions: What does it mean to quantify emotion? Is it possible to detect emotions in data? Which sensing modalities are the most suitable for accurately detecting emotions? What are issues of interpreting emotionsensing data?

- Approaches to detecting emotions: How can we collect meaningful data? What novel approaches are out there?
- The quest for an accurate ground-truth: A major issue of all research on emotions is the subjectivity of emotional states. How can we ensure that ground-truth is reliable, comparable, and generalizable?

- Tools and datasets: How reliable are available emotion detection systems, tools, and algorithms? What renders these feasible or non-feasible? Where are gaps for improvement?

- Application cases: What are concrete cases of systems that effectively detect emotions? What applications, technologies, and systems support the mental health of their users? Where are gaps and chances in those systems?

- Risks of emotion detection: Should our aim be merely to achieve more accurate emotion detection? What are ethical and privacy concerns?

- The emerging field of Digital Emotion Regulation: What do we do, once we have quantified emotions? How can we use technology to influence emotional trajectories, and create intervention systems that support emotional wellbeing?

\section{WORKSHOP STRUCTURE}

We propose a 4-hour virtual workshop. We will use a combination of ZOOM and Miro to keep our participants engaged and record the workshop findings. In the first quarter the organizers will introduce the workshop theme, and two keynote speakers will give talks. Presentation sessions will be distributed across the second, third, and fourth quarter of the workshop. We will initiate group discussions on fundamental challenges and prepare a group activity to keep participants engaged and foster collaboration. We will also leave room for demo presentations.

\subsection{Participants}

We plan for 15-20 participants - excluding organizers - to participate in the workshop. The paper presentations will be curated and organized in themes by the organizers. We will put special focus on including contributions from different research fields in one session to stimulate fruitful discussions.

\subsection{Keynotes}

We will kick off the workshop with two prominent researchers from the fields of Psychology (Maya Tamir) and HCI (Rafael A. Calvo).

\subsection{Presentations and Demonstrations}

The organization committee will call for participation through established mailing lists, and in the networks of the organizers. All submissions will be peer-reviewed and included in the ACM Digital Library and supplemental proceedings of the conference. Workshop organizers must clearly describe in their proposal whether they plan to have workshop contributions published in this way. Organizers. We will curate and invite accepted submissions for oral presentations. We will put special attention on sufficient discussion times and ensure that there is a time window available for participants to demo technologies and prototypes. 


\subsection{Action Groups}

We will create action groups, consisting of 5-6 members each, that will have to develop short 1-year, 3-year, 5-year, and 10-year research plans on one of the seven research themes of the workshop. All groups will present their plans, and the solutions and proposals will be collected in a shared visual management tool, e.g., Miro.

\subsection{Group Discussion}

Following the action groups, we will actively engage with the audience to discuss the findings. In conclusion, we will summarize the key points and develop a research agenda, which is later to be shared with all workshop participants in a call for cross-disciplinary collaboration.

\section{POST WORKSHOP PLANS}

Based on the activities, Miro-board notes, findings, and discussions, the organizers will synthesize the findings of the action groups and discussions and will disseminate the outcomes to the broader community through the workshop website. The organizers will summarize the contributions and outcomes of the workshop and will develop a research agenda on "Making Sense of Emotion-Sensing: How to Quantify Human Emotions." This research agenda will be distributed to all participants in a targeted call for collaboration and submitted to the ACM Interactions magazine for wider dissemination.

\section{ACKNOWLEDGMENTS}

This work is partially funded by the Australian Research Council Discovery Project, ARC-DP190102627.

\section{REFERENCES}

[1] Ebrahim Babaei, Benjamin Tag, Tilman Dingler, and Eduardo Velloso. 2021. A Critique of Electrodermal Activity Practices at CHI. In Proceedings of the 2021 CHI Conference on Human Factors in Computing Systems. ACM, New York, NY, USA, 1-14. https://doi.org/10.1145/3411764.3445370

[2] Lisa Feldman Barrett. 2017. The theory of constructed emotion: an active inference account of interoception and categorization. Social cognitive and affective neuroscience 12, 1 (2017), 1-23. https://doi.org/10.1093/scan/nsw154

[3] Lisa Feldman Barrett, Ralph Adolphs, Stacy Marsella, Aleix M. Martinez, and Seth D. Pollak. 2019. Emotional Expressions Reconsidered: Challenges to Inferring Emotion From Human Facial Movements. Psychological Science in the Public Interest 20, 1 (2019), 1-68. https://doi.org/10.1177/1529100619832930

[4] Shaun Canavan, Marvin Andujar, Lijun Yin, Anton Nijholt, and Elizabeth Schotter. 2018. Ubiquitous emotion recognition with multimodal mobile interfaces. UbiComp/ISWC 2018 Adjunct (2018), 937-941. https://doi.org/10.1145/3267305. 3274139

[5] Larry Chan, Vedant Das Swain, Christina Kelley, Kaya de Barbaro, Gregory D Abowd, and Lauren Wilcox. 2018. Students' Experiences with Ecological Momentary Assessment Tools to Report on Emotional Well-being. Proceedings of the ACM on Interactive, Mobile, Wearable and Ubiquitous Technologies 2, 1 (2018), 1-20. https://doi.org/10.1145/3191735

[6] Dick Clomén, Rebecca Horn, Milena Osorio, Melanie Powell, Anne Faigh Rydell, and Zara Sejberg. 2020. The importance of mental health and psychosocial support during COVID-19. Technical Report. International Federation of Red Cross and Red Crescent Societies and International Committee of the Red Cross. https: //www.icrc.org/en/document/crisis-mental-health-COVID-19

[7] Desirée Colombo, Javier Fernández-álvarez, Azucena García Palacios, Pietro Cipresso, Cristina Botella, and Giuseppe Riva. 2019. New technologies for the understanding, assessment, and intervention of emotion regulation. Frontiers in Psychology 10, JUN (2019). https://doi.org/10.3389/fpsyg.2019.01261

[8] Sylvain Delplanque and David Sander. 2021. A fascinating but risky case of reverse inference: From measures to emotions! Food Quality and Preference November 2020 (2021), 104183. https://doi.org/10.1016/j.foodqual.2021.104183
[9] Anja Exler, Christoph Klebsattel, Andrea Schankin, and Michael Beigl. 2016. A wearable system for mood assessment considering smartphone features and data from mobile ECGs. UbiComp 2016 Adjunct (2016), 1153-1161. https://doi.org/10. $1145 / 2968219.2968302$

[10] B. Figner and R. O. Murphy. 2011. Using skin conductance in judgment and decision making research. ... Tracing Methods for Decision Research January 2011 (2011), 1-33.

[11] Jorge Goncalves, Pratyush Pandab, Denzil Ferreira, Mohammad Ghahramani, Guoying Zhao, and Vassilis Kostakos. 2014. Projective Testing of Diurnal Collective Emotion. In Proceedings of the 2014 ACM International Foint Conference on Pervasive and Ubiquitous Computing (Seattle, Washington) (UbiComp '14). 487-497. https://doi.org/10.1145/2632048.2636067

[12] Xin Hu, Jingjing Chen, Fei Wang, and Dan Zhang. 2019. Ten challenges for EEG-based affective computing. Brain Science Advances 5, 1 (2019), 1-20. https: //doi.org/10.1177/2096595819896200

[13] Soumy Jacob, Andreas Dengel, and Shoya Ishimaru. 2018. Poster: Interest detection while reading newspaper articles by utilizing a physiological sensing wristband. UbiComp/ISWC 2018 Adjunct (2018), 78-81. https://doi.org/10.1145/ 3267305.3267666

[14] Rahatul Jannat, Iyonna Tynes, Juan Adorno, Shaun Canavan, and Lott LaLime. 2018. Ubiquitous emotion recognition using audio and video data. UbiComp/ISWC 2018 Adjunct (2018), 956-959. https://doi.org/10.1145/3267305.3267689

[15] Matthew D. Lieberman. 2019. Boo! The consciousness problem in emotion. Cognition and Emotion 33, 1 (2019), 24-30. https://doi.org/10.1080/02699931.2018. 1515726

[16] Martin Maritsch, Caterina Bérubé, Mathias Kraus, Vera Lehmann, Thomas Züger, Stefan Feuerriegel, Tobias Kowatsch, and Felix Wortmann. 2019. Improving heart rate variability measurements from consumer smartwatches with machine learning. UbiComp/ISWC 2019 Adjunct (2019), 934-938. https://doi.org/10.1145/ 3341162.3346276 arXiv:1907.07496

[17] Shivsevak Negi and Ritayan Mitra. 2018. Poster: EEG metrics to determine cognitive load and affective states - A pilot study. UbiComp/ISWC 2018 (2018), 182-185. https://doi.org/10.1145/3267305.3267618

[18] Rosalind W Picard. 1997. Affective Computing. MIT Press, Cambridge, MA, USA

[19] Zhanna Sarsenbayeva, Gabriele Marini, Niels van Berkel, Chu Luo, Weiwei Jiang, Kangning Yang, Greg Wadley, Tilman Dingler, Vassilis Kostakos, and Jorge Goncalves. 2020. Does smartphone use drive our emotions or vice versa? A causal analysis. In Proceedings of the ACM SIGCHI Conference on $\mathrm{Hu}$ man Factors in Computing Systems 2020 (CHI'20). ACM, New York, 36:1-36:15. https://doi.org/10.1145/3313831.3376163

[20] Zhanna Sarsenbayeva, Benjamin Tag, Shu Yan, Vassilis Kostakos, and Jorge Goncalves. 2020. Using Video Games to Regulate Emotions. In 32nd Australian Conference on Human-Computer Interaction. ACM, New York, NY, USA, 755-759. https://doi.org/10.1145/3441000.3441035

[21] Luke Stark and Jesse Hoey. 2021. The Ethics of Emotion in Artificial Intelligence Systems. In Proceedings of the 2021 ACM Conference on Fairness, Accountability, and Transparency, Vol. 2019. ACM, New York, NY, USA, 782-793. https://doi. org $/ 10.1145 / 3442188.3445939$

[22] Benjamin Tag, Christian Holz, Paul Lukowicz, Olivier Augereau, Yuji Uema, and Kai Kunze. 2018. EyeWear 2018: Second Workshop on EyeWear Computing. UbiComp/ISWC 2018 Adjunct (2018), 964-967. https://doi.org/10.1145/3267305. 3274140

[23] Benjamin Tag, Jamie A. Ward, Yuji Uema, and Kai Kunze. 2019. Eyewear 2019: Third workshop on eyewear computing - Focus: Social interactions. UbiComp/ISWC 2019 Adjunct (2019), 616-618. https://doi.org/10.1145/3341162. 3347761

[24] Aku Visuri, Zhanna Sarsenbayeva, Jorge Goncalves, Evangelos Karapanos, and Simon Jones. 2016. Impact of Mood Changes on Application Selection. In Proceedings of the 2016 ACM International foint Conference on Pervasive and Ubiquitous Computing: Adjunct (Heidelberg, Germany) (UbiComp '16). Association for Computing Machinery, New York, NY, USA, 535-540. https://doi.org/10.1145/2968219. 2968317

[25] World Health Organization. 2020. COVID-19 disrupting mental health services in most countries, WHO survey. https://www.who.int/news/item/05-10-2020covid-19-disrupting-mental-health-services-in-most-countries-who-survey

[26] Yuki Yamada, Dominik Borna Ćepulić, Tao Coll-Martín, Stéphane Debove, Guillaume Gautreau, Hyemin Han, Jesper Rasmussen, Thao P. Tran, Giovanni A. Travaglino, COVIDiSTRESS Global Survey Consortium, and Andreas Lieberoth. 2021. COVIDiSTRESS Global Survey dataset on psychological and behavioural consequences of the COVID-19 outbreak. Scientific Data 8, 1 (2021), 1-23. https://doi.org/10.1038/s41597-020-00784-9

[27] Kangning Yang, Chaofan Wang, Zhanna Sarsenbayeva, Benjamin Tag, Tilman Dingler, Greg Wadley, and Jorge Goncalves. 2020. Benchmarking commercial emotion detection systems using realistic distortions of facial image datasets. The Visual Computer (2020), 1-20. https://doi.org/10.1007/s00371-020-01881-x 